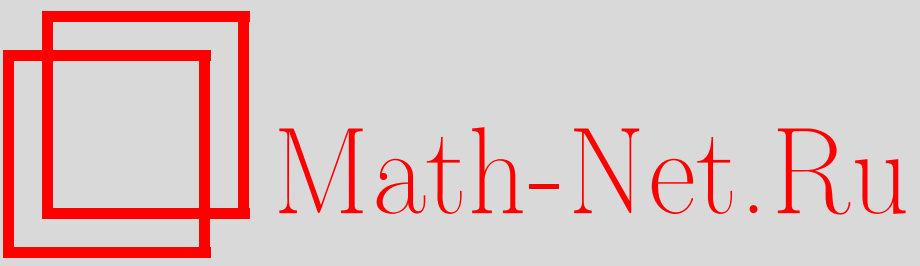

А. В. Кулешов, Линейные реперы как орбиты проективных реперов, Итоги науки и техн. Сер. Соврем. мат. и ее прил. Темат. обз., 2020, том 180, 58-65

DOI: https://doi.org/10.36535/0233-6723-2020-180-58-65

Использование Общероссийского математического портала Math-Net.Ru подразумевает, что вы прочитали и согласны с пользовательским соглашением

http://www.mathnet.ru/rus/agreement

Параметры загрузки:

IP : 3.82 .47 .9

26 апреля 2023 г., 10:02:36

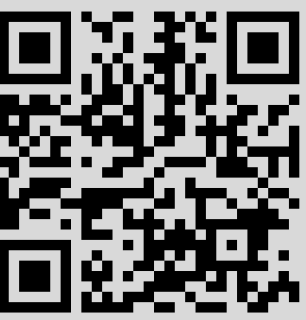


ИТОГИ НАУКИ И ТЕХНИКИ.

Современная математика и ее приложения.

Тематические обзоры.

Том 180 (2020). C. $58-65$

DOI: $10.36535 / 0233-6723-2020-180-58-65$

УДК 514.75

\title{
ЛИНЕЙНЫЕ РЕПЕРЫ КАК ОРБИТЫ ПРОЕКТИВНЫХ РЕПЕРОВ
}

\author{
(C) 2020 г. $\quad$ А. В. КУЛЕШОВ
}

\begin{abstract}
АннотАция. Рассматривается многомерное проективное пространство с выделенной точкой (центром). На многообразии проективных реперов данного пространства, адаптированных центру, определено действие стабилизатора центра в группе проективных преобразований. Показано, что линейные реперы, т.е. базисы касательного векторного пространства к проективному пространству в его центре, можно отождествить с орбитами адаптированных проективных реперов по действию ядра эпиморфизма групп Ли, сопоставляющего каждому преобразованию из стабилизатора его дифференциал в центре. При помощи многомерного обобщения теоремы Дезарга получен критерий принадлежности двух адаптированных проективных реперов одной орбите.
\end{abstract}

Ключевые слова: проективное пространство, проективный репер, линейный репер, пространство орбит, теорема Дезарга.

\section{LINEAR FRAMES AS ORBITS OF PROJECTIVE FRAMES}

\author{
(c) 2020 A. V. KULESHOV
}

\begin{abstract}
A multidimensional projective space with a marked point (center) is considered. On the manifold of projective frames of the given space that are adapted to the center, the action of the stabilizer of the center of the group of projective transformations is defined. We prove that linear frames, i.e., bases of the tangent vector space of the projective space at its center, can be identified with orbits of the adapted projective frames with respect to the action of the kernel of the epimorphism of Lie groups, which assigns to each transformation from the stabilizer its differential at the center. Using a multidimensional generalization of the Desargues theorem, we obtain a criterion for two adapted projective frames to belong to the same orbit.
\end{abstract}

Keywords and phrases: projective space, projective frame, linear frame, orbit space, Desargues theorem.

AMS Subject Classification: 53A20, 14N20

1. Введение. Метод внешних дифференциальных форм и подвижного репера, разработанный Э. Картаном в первой половине XX в. (см., например, $[9,11])$, успешно применяется при изучении однородных пространств и пространств со связностями и, в частности, в задачах локальной проективной дифференциальной геометрии подмногообразий (см. монографию [7]). В рамках данного метода с подмногообразием однородного пространства ассоциируется главное расслоение реперов, адаптированных данному подмногообразию, и дальнейшее исследование производится в терминах так называемых структурных уравнений, т.е. уравнений на внешние дифференциалы базисных и слоевых форм данного расслоения. Анализируя систему структурных уравнений, можно, в частности, установить существование главных фактор-расслоений данного расслоения. Однако описание явной конструкции таких фактор-расслоений является самостоятельной задачей, зачастую нетривиальной. Ее решение основано на следующем принципе: точки фактор- 
расслоения суть орбиты точек исходного расслоения по действию некоторой подгруппь структурной группы последнего. Таким образом, понять, как устроены такие орбиты не только с топологической, но также и с геометрической точки зрения - первый шаг на пути решения данной задачи.

В частности, в ряде работ (см., например, $[1,5,6])$ рассматривается главное расслоение центропроективных реперов над различного вида гладкими семействами плоскостных элементов в проективном пространстве. Исходя из вида структурных уравнений данного расслоения, авторы заключают о наличии главного фактор-расслоения, структурная группа которого изоморфна полной линейной группе, не описывая элементы такого фактор-расслоения явным образом. Цель настоящей работы - восполнить указанный пробел, что в свете сформулированного принципа означает изучение геометрического устройства орбит проективных реперов по действию подходящей нормальной подгруппы $H$ структурной группы $G$ исходного расслоения. Ввиду того, что в проективной геометрии реперы приобретают наглядный геометрический смысл, естественно поставить вопрос о критерии принадлежности двух реперов одной $H$-орбите в бескоординатной форме, т.е. в виде геометрической конструкции в духе синтетической геометрии. Заметим, что для достижения поставленной цели достаточно ограничиться одним отдельно взятым слоем рассматриваемого расслоения.

Кратко опишем структуру и результаты настоящей работы. В разделе 2 рассматривается $n$ мерное проективное пространство $\mathbb{P}_{n}$ вместе с выделенной точкой $A \in \mathbb{P}_{n}$. На многообразии $\mathcal{F}\left(\mathbb{P}_{n}\right)$ всех адаптированных проективных реперов пространства $\mathbb{P}_{n}$ (т.е. проективных реперов с первой вершиной $A$ ) естественным образом задается левое действие стабилизатора $G$ точки $A$ в группе проективных преобразований $G P(n)$ пространства $\mathbb{P}_{n}$. Аналогично, на многообразии $\mathcal{F}(V)$ всех базисов касательного векторного пространства $V=T_{A} \mathbb{P}_{n}$ к $\mathbb{P}_{n}$ в точке $A$ определено левое действие группы $\mathbf{G L}(V)$ невырожденных эндоморфизмов пространства $V$. Нормальный делитель $H$ группы $G$ определяется как ядро эпиморфизма групп Ли $\beta: G \rightarrow \mathbf{G L}(V), g \mapsto d_{A} g$ (раздел 3 ). При этом факторгруппа $G / H$ канонически изоморфна группе $\mathbf{G L}(V)$, их можно отождествить и обозначить общим символом $\bar{G}$. На пространстве $H$-орбит $\mathcal{Q}\left(\mathbb{P}_{n}\right)$ корректно определяется правое действие факторгруппы $\bar{G}$, причем $\mathcal{Q}\left(\mathbb{P}_{n}\right)$ и $\mathcal{F}(V)$ канонически изоморфны как $\bar{G}$-пространства (теорема 1). Таким образом, базисы (линейные реперы) касательного пространства $V$ можно трактовать как $H$-орбиты адаптированных проективных реперов.

В разделе 4 показано, что необходимым условием эквивалентности (т.е. принадлежности одной $H$-орбите) двух адаптированных реперов является их перспективность (теорема 2). Однако это условие не является достаточным, и с целью уточнения геометрической характеристики отношения эквивалентности вводится в рассмотрение отношение строгой перспективности. Для двух строго перспективных реперов имеет место $n$-мерное обобщение теоремы Дезарга (теорема 3 ), в соответствии с которым при $n \geqslant 2$ определена гиперплоскость Дезарга $\mathcal{L}_{\left(\mathcal{R}, \mathcal{R}^{\prime}\right)}$. Основной результат данной работы состоит в следующем: при $n \geqslant 2$ два строго перспективных репера эквивалентны тогда и только тогда, когда порожденная ими гиперплоскость Дезарга проходит через центр $A$ (теорема 4). Таким образом, получено искомое геометрическое описание таких $H$ орбит, не привлекающее образы касательного пространства $V$.

Наконец, в разделе 5 описаны действия матричных групп на пространствах $\mathcal{F}\left(\mathbb{P}_{n}\right), \mathcal{Q}\left(\mathbb{P}_{n}\right)$ и $\mathcal{F}(V)$, и показано, что $\mathcal{Q}\left(\mathbb{P}_{n}\right)$ и $\mathcal{F}(V)$ изоморфны как $\mathbf{G L}(n)$-пространства.

2. Основные понятия и утверждения. Пусть $V_{n+1}$ - векторное пространство размерности $(n+1)$ над полем $\mathbb{R}$ или $\mathbb{C}$, и пусть через обозначено отношение коллинеарности на $V_{n+1}^{0}=V_{n+1} \backslash\{\mathbf{0}\}$. Тогда под $n$-мерным проективным пространством $\mathbb{P}_{n}$, как и в [2], будем понимать факторпространство $\mathbb{P}_{n}=V_{n+1}^{0} / \sim$. При этом векторное пространство $V_{n+1}$ будем называть ассоциированным с $\mathbb{P}_{n}$, а отображение $\pi: V_{n+1}^{0} \rightarrow \mathbb{P}_{n}$, сопоставляющее каждому вектору $\boldsymbol{a} \in V_{n+1}^{0}$ его класс эквивалентности $[\boldsymbol{a}]$, назовем канонической сюръекцией.

Отображение $g: \mathbb{P}_{n} \rightarrow \mathbb{P}_{n}$ называется проективным преобразованием, если найдется невырожденный линейный оператор $\tilde{g}: V_{n+1} \rightarrow V_{n+1}$ такой, что $\pi \circ \tilde{g}_{0}=g \circ \pi$, где $\tilde{g}_{0}-$ сужение $\tilde{g}$ на $V_{n+1}^{0}$. При этом $\tilde{g}$ будем называть оператором, накрывающим $g$. Группу проективных преобразований пространства $\mathbb{P}_{n}$ обозначим через $G P(n)$. 
Проективный репер в $\mathbb{P}_{n}$ - это упорядоченный набор $\mathcal{R}$ из $n+2$ точек $A_{0}, A_{1}, \ldots, A_{n}, E \in \mathbb{P}_{n}$ таких, что любая совокупность из $n+1$ точек этого набора находится в общем положении:

$$
\mathcal{R}=\left\{A_{0}, A_{1}, \ldots, A_{n}, E\right\} .
$$

Замечание 1. Здесь и далее элементы упорядоченных наборов заключаются в фигурные скобки.

Говорят, что базис

$$
\mathcal{R}=\left\{\boldsymbol{A}_{\mathbf{0}}, \boldsymbol{A}_{1}, \ldots, \boldsymbol{A}_{n}\right\}
$$

пространства $V_{n+1}$ порождает репер (1), если

$$
\begin{gathered}
\pi\left(\boldsymbol{A}_{0}\right)=A_{0}, \quad \pi\left(\boldsymbol{A}_{1}\right)=A_{1}, \quad \ldots, \quad \pi\left(\boldsymbol{A}_{n}\right)=A_{n}, \\
\pi\left(\boldsymbol{A}_{0}+\boldsymbol{A}_{1}+\ldots+\boldsymbol{A}_{n}\right)=E .
\end{gathered}
$$

Однородными координатами точки $M$ относительно репера $\mathcal{R}$ называются координаты некоторого вектора $\boldsymbol{M} \in \pi^{-1}(M)$ относительно базиса $\mathcal{R}$, порождающего этот репер $\mathcal{R}$ :

$$
M\left(x^{0}: x^{1}: \ldots: x^{n}\right)_{\mathcal{R}} \Longleftrightarrow \boldsymbol{M}=x^{0} \boldsymbol{A}_{0}+x^{1} \boldsymbol{A}_{1}+\ldots+x^{n} \boldsymbol{A}_{n} .
$$

Через $U_{\mathcal{R}}$ обозначим подмножество пространства $\mathbb{P}_{n}$, задаваемое уравнением $x^{0} \neq 0$. Аффинными (неоднородными) координатами точки $M \in U_{\mathcal{R}}$ относительно репера $\mathcal{R}$ называются отношения:

$$
X^{i}=\frac{x^{i}}{x^{0}}, \quad i=\overline{1, n}
$$

Заметим, что $U_{\mathcal{R}}$ является открытой окрестностью центра $A$.

Аффинной картой в репере $\mathcal{R}$ называется локальная карта $\left(U_{\mathcal{R}}, \varphi_{\mathcal{R}}\right)$, где $\varphi_{\mathcal{R}}$ - отображение, сопоставляющее каждой точке $M \in U_{\mathcal{R}}$ набор ее неоднородных координат относительно данного репера:

$$
\varphi_{\mathcal{R}}: U_{\mathcal{R}} \rightarrow \mathbb{R}^{n}, \quad M \mapsto\left(X^{1} \ldots, X^{n}\right) .
$$

Определение 1 . Выделим некоторую точку $A \in \mathbb{P}_{n}$ и назовем ее центром пространства $\mathbb{P}_{n}$. Назовем проективный репер (1) адаптированным (центропроективным), если его первая вершина $A_{0}$ совпадает с центром, т.е. $A_{0}=A$. Множество всех адаптированных проективных реперов обозначим через $\mathcal{F}\left(\mathbb{P}_{n}\right)$. Стабилизатор точки $A$ в группе $G P(n)$ обозначим через $G$.

Пусть $\mathcal{R}$ и $\mathcal{R}^{\prime}$ - адаптированные реперы. Тогда существует единственный набор коэффициентов $\alpha_{i}, \alpha_{j}^{i}$ таких, что для любой точки $M \in U_{\mathcal{R}} \cap U_{\mathcal{R}^{\prime}}$ справедливы равенства

$$
X^{i}=\frac{\alpha_{j}^{i} \tilde{X}^{j}}{1+\alpha_{j} \tilde{X}^{j}}, \quad \operatorname{det}\left(\alpha_{j}^{i}\right) \neq 0
$$

где $X^{i}$ и $\tilde{X}^{i}$ - аффинные координаты точки $M$ относительно реперов $\mathcal{R}$ и $\mathcal{R}^{\prime}$ соответственно. Уравнения (4) назовем формулами перехода от репера $\mathcal{R}$ к реперу $\mathcal{R}^{\prime}$.

Любой линейный оператор $\tilde{g}$, накрывающий преобразование $g \in G$, корректно определяет оператор

$$
\tilde{g}^{\sharp}: V_{n+1} / \hat{A} \rightarrow V_{n+1} / \hat{A},
$$

такой, что

$$
\tilde{g}^{\sharp}(\boldsymbol{M}+\hat{A})=\tilde{g}(\boldsymbol{M})+\hat{A}, \quad \boldsymbol{M} \in V_{n+1},
$$

где $\hat{A}$ - линейная оболочка прообраза $\pi^{-1}(A)$ в пространстве $V_{n+1}$.

Пусть $V$ - касательное векторное пространство $T_{A} \mathbb{P}_{n}$ к $\mathbb{P}_{n}$ в точке $A$. Имеет место

Лемма 1 (см. [11]). Существует канонический изоморфизм векторных пространств

$$
T_{A} \mathbb{P}_{n} \cong \hat{A}^{*} \otimes\left(V_{n+1} / \hat{A}\right) .
$$


В дальнейшем каждый вектор $\boldsymbol{\xi}$ пространства $V$ будем отождествлять с линейным оператором $\xi: \hat{A} \rightarrow V_{n+1} / \hat{A}$ соответствующим вектору $\boldsymbol{\xi}$ при данном изоморфизме; при этом для любого преобразования $g \in G$ его дифференциал $d_{A} g: V \rightarrow V$ можно интерпретировать как оператор, действующий по закону композиции отображений

$$
d_{A} g: \xi \mapsto \tilde{g}^{\sharp} \circ \xi,
$$

где $\tilde{g}$ - такой оператор, накрывающий $g$, что $\tilde{g}(\boldsymbol{A})=\boldsymbol{A}$ для некоторого $\boldsymbol{A} \in \pi^{-1}(A)$.

Хорошо известно, что каждая локальная карта на произвольном гладком многообразии порождает поле натурального базиса в точках области данной карты. В случае аффинной карты (3) такой базис может быть интерпретирован следующим образом:

Утверждение 1. Для любого адаптированного репера (1) найдется единственный базис

$$
\mathcal{B}_{\mathcal{R}}=\left\{\xi_{1}, \ldots, \xi_{n}\right\}
$$

касательного пространства $V$ такой, что для некоторого (и, как следствие, любого) базиса (2), порождающего данный репер, справедливы равенства

$$
\xi_{i}\left(\boldsymbol{A}_{0}\right)=\boldsymbol{A}_{i}+\hat{A}, \quad i=\overline{1, n} .
$$

Через $\mathcal{F}(V)$ обозначим множество всех базисов пространства $V$, а через $\mathbf{G L}(V)$ - группу невырожденных линейных операторов, действующих на $V$.

Кроме того, для произвольного множества $X$ обозначим через $S(X)$ группу биекций данного множества на себя.

Следующие отображения являются корректно определенными свободными транзитивными представлениями групп Ли $G$ и $\mathbf{G L}(V)$, действующих слева соответственно на многообразиях $\mathcal{F}\left(\mathbb{P}_{n}\right)$ и $\mathcal{F}(V)$ :

$$
\begin{gathered}
\Phi: G \rightarrow S\left(\mathcal{F}\left(\mathbb{P}_{n}\right)\right), \quad \Psi: \mathbf{G L}(V) \rightarrow S(\mathcal{F}(V)), \\
\Phi_{g}(\mathcal{R}):=g \cdot \mathcal{R}=\left\{g\left(A_{0}\right), g\left(A_{1}\right), \ldots, g\left(A_{n}\right), g(E)\right\}, \quad g \in G ; \\
\Psi_{\rho}(\mathcal{B}):=\rho \cdot \mathcal{B}=\left\{\rho\left(\boldsymbol{\xi}_{1}\right), \ldots, \rho\left(\boldsymbol{\xi}_{n}\right)\right\}, \quad \rho \in \mathbf{G L}(V),
\end{gathered}
$$

где

$$
\mathcal{R}=\left\{A_{0}, A_{1}, \ldots, A_{n}, E\right\}, \quad \mathcal{B}=\left\{\boldsymbol{\xi}_{1}, \ldots, \boldsymbol{\xi}_{n}\right\} .
$$

Определение 2. Пусть $g \in G, \mathcal{R} \in \mathcal{F}\left(\mathbb{P}_{n}\right)$. Уравнениями преобразования $g$ относительно репера $\mathcal{R}$ назовем формулы перехода от репера $\mathcal{R}$ к реперу $g . \mathcal{R}$.

\section{3. Пространство $H$-орбит.}

Лемма 2 (см. [10]). Пусть $\varphi: G \rightarrow G^{\prime}$-непрерывный гомоморфизм групп Ли $и H=\operatorname{ker} \varphi$. Тогда $H$-нормальный делитель группы Ли $G$, а $G / H$ - группа Ли; при этом индуцированное отображение $\bar{\varphi}: G / H \rightarrow G^{\prime}$ есть ингективная иммерсия этой группы Ли в $G^{\prime}$.

В силу свойств дифференциала отображения и леммы 2 справедливо

Утверждение 2. Отображение

$$
\beta: G \rightarrow \mathbf{G L}(V), \quad g \mapsto d_{A} g
$$

является эпиморфизмом групп Ли, причем ядро $H=\operatorname{ker} \beta$ является замкнутой нормальной подгруппой в $G$, а факторгруппа $G / H$ канонически изоморфна группе $\mathbf{G L}(V)$ :

$$
\bar{\beta}: G / H \rightarrow \mathbf{G L}(V), \quad g H \mapsto d_{A} g .
$$

Утверждение 2 позволяет нам отождествить группы Ли $G / H$ и $\mathbf{G L}(V)$.

Определение 3. Группа Ли $\bar{G}:=G / H=\mathbf{G L}(V)$ называется линейной факторгруппой группы $G$.

Пусть $g \in G, \mathcal{R} \in F\left(\mathbb{P}_{n}\right)$, и пусть (4) суть уравнения преобразования $g$ относительно $\mathcal{R}$. Тогда

$$
g \in H \quad \Longleftrightarrow \quad \alpha_{j}^{i}=\delta_{j}^{i} \quad \Longleftrightarrow \quad X^{i}=\frac{\tilde{X}^{i}}{1+\alpha_{j} \tilde{X}^{j}} .
$$


Определение 4. Два адаптированных репера $\mathcal{R}$ и $\mathcal{R}^{\prime}$ назовем эквивалентными $\left(\mathcal{R} \sim \mathcal{R}^{\prime}\right)$, если они принадлежат одной $H$-орбите. Обозначим через $[\mathcal{R}]$ класс эквивалентности репера $\mathcal{R}$, через $\mathcal{Q}\left(P_{n}\right)$ - множество всех таких классов эквивалентности, и через $p: F\left(\mathbb{P}_{n}\right) \rightarrow \mathcal{Q}\left(\mathbb{P}_{n}\right)$ обозначим каноническую проекцию $\mathcal{R} \mapsto[\mathcal{R}]$.

Каждое действие $G$ на $\mathcal{F}\left(\mathbb{P}_{n}\right)$ отображает $H$-орбиты в $H$-орбиты, причем любые два элемента $g_{1}, g_{2} \in G$, принадлежащие одному и тому же смежному классу по $H$, действуют на пространстве $H$-орбит $\mathcal{Q}\left(\mathbb{P}_{n}\right)$ одинаковым образом. Тем самым получено

На пространстве $H$-орбит $\mathcal{Q}\left(P_{n}\right)$ корректно определено свободное транзитивное действие факторгруппы $\bar{G}$ слева:

$$
\bar{\Psi}: \bar{G} \rightarrow S\left(\mathcal{Q}\left(\mathbb{P}_{n}\right)\right), \quad \bar{\Psi}_{g H}([\mathcal{R}])=g H .[\mathcal{R}]=[g \cdot \mathcal{R}], \quad g \in G, \mathcal{R} \in \mathcal{F}\left(\mathbb{P}_{n}\right) .
$$

Утверждение 3. Рассмотрим отображение

$$
\alpha: \mathcal{F}\left(\mathbb{P}_{n}\right) \rightarrow \mathcal{F}(V), \quad \alpha: \mathcal{R} \mapsto \mathcal{B}_{\mathcal{R}},
$$

где базис $\boldsymbol{B}_{\mathcal{R}}$ определен однозначно в силу утверждения 1.

1. Для любого $g \in G$ справедливо равенство

$$
\alpha \circ g=\Psi_{d_{A} g} \circ \alpha .
$$

2. $\alpha$-сюрвективная субмерсия.

3. Н-Орбиты реперов суть в точности полные прообразы базисов пространства $V$ относительно отображения $\alpha$, т.е.

4. Корректно определено отображение

$$
\mathcal{R} \sim \mathcal{R}^{\prime} \quad \Longleftrightarrow \quad \alpha(\mathcal{R})=\alpha\left(\mathcal{R}^{\prime}\right) .
$$

$$
\bar{\alpha}: \mathcal{Q}\left(\mathbb{P}_{n}\right) \rightarrow F(V), \quad[\mathcal{R}] \mapsto \mathcal{B}_{\mathcal{R}}
$$

Теорема 1. $\bar{\alpha}: \mathcal{Q}\left(\mathbb{P}_{n}\right) \rightarrow \mathcal{F}(V)$ - изоморфизм $\bar{G}$-пространств.

Доказательство. Теорема 1 вытекает из коммутативности следующей диаграммы для любого $g \in G:$

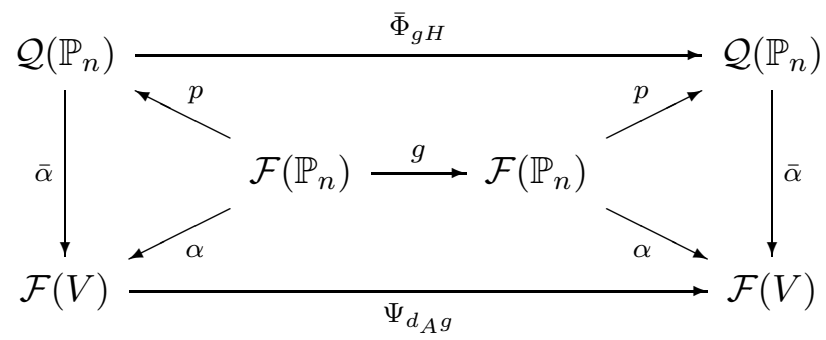

4. Геометрическое описание $H$-орбит. Далее ограничимся случаем $n \geqslant 2$. Пусть $\mathcal{R}, \mathcal{R}^{\prime} \in$ $\mathcal{F}\left(\mathbb{P}_{n}\right)$, где

$$
\mathcal{R}=\left\{A, A_{1}, \ldots, A_{n}, E\right\}, \quad \mathcal{R}^{\prime}=\left\{A, A_{1}^{\prime}, \ldots, A_{n}^{\prime}, E^{\prime}\right\} .
$$

Определение 5. Два репера $\mathcal{R}$ и $\mathcal{R}^{\prime}$ называются перспективными $\left(\mathcal{R} \cong \mathcal{R}^{\prime}\right)$, если

$$
A_{i}^{\prime} \in A_{i} A_{0} \quad(i=\overline{1, n}), \quad E^{\prime} \in E A_{0},
$$

где, например, $E A_{0}$ - прямая, проходящая через точку $E$ и центр $A_{0}$.

Утверждение 4. Пусть $\mathcal{R}$ и $\mathcal{R}^{\prime}$ - произвольные адаптированные реперы. Тогда $\mathcal{R}$ и $\mathcal{R}^{\prime}$ перспективны в том и только в том случае, если формулы перехода от $\mathcal{R} \kappa \mathcal{R}^{\prime}$ имеют вид

$$
X^{i}=\frac{h \tilde{X}^{i}}{1+a_{j} \tilde{X}^{j}}, \quad i=\overline{1, n},
$$

для некоторых коэффициентов $h \neq 0, a_{1}, \ldots, a_{n}$.

Замечание 2. В частном случае, когда все коэффициенты $a_{i}$ равны $0(i=\overline{1, n})$, преобразование (6) является гомотетией в аффинной карте $\left(\varphi_{\mathcal{R}}, U_{\mathcal{R}}\right)$. 
Непосредственно из (6) и (5) вытекает следующая теорема.

Теорема 2. Два адаптированных проективных репера $\mathcal{R}$ и $\mathcal{R}^{\prime}$ эквивалентны тогда и только тогда, когда, во-первых, они перспективны, и, во-вторых, коэфбициент $h$ (см. формулу (6)) равен единище:

$$
\mathcal{R} \sim \mathcal{R}^{\prime} \quad \Longleftrightarrow \quad\left\{\begin{array}{c}
\mathcal{R} \cong \mathcal{R}^{\prime}, \\
h=1 .
\end{array}\right.
$$

Определение 6. Назовем реперы $\mathcal{R}$ и $\mathcal{R}^{\prime}$ строго перспективными, если они перспективны и их соответствующие точки не совпадают, т.е.

$$
A_{i}^{\prime} \neq A_{i} \quad(i=\overline{1, n}), \quad E^{\prime} \neq E .
$$

Определение 7. Для любого набора подмножеств $Y_{1}, \ldots, Y_{s} \subset \mathbb{P}_{n}$ обозначим через $\left\langle Y_{1}, \ldots, Y_{s}\right\rangle$ наименьшую (по включению) плоскость пространства $\mathbb{P}_{n}$, содержащую все данные подмножества.

Далее введем следующие обозначения:

$$
\mathcal{M}:=\left\langle A_{1}, \ldots A_{n}\right\rangle, \quad \mathcal{M}^{\prime}:=\left\langle A_{1}^{\prime}, \ldots A_{n}^{\prime}\right\rangle, \quad \mathcal{N}=\mathcal{M} \cap \mathcal{M}^{\prime} .
$$

Для двух строго перспективных реперов $\mathcal{R}$ и $\mathcal{R}^{\prime}$ определены точки

$$
B_{i}=A_{i} E \cap A_{i}^{\prime} E^{\prime}, \quad i=\overline{1, n},
$$

и плоскость

$$
\mathcal{L}_{\left(\mathcal{R}, \mathcal{R}^{\prime}\right)}:=\left\langle B_{1}, \ldots B_{n}, \mathcal{N}\right\rangle .
$$

Кроме того, в данном случае $\mathcal{M}$ и $\mathcal{M}^{\prime}$ не совпадают, и поэтому $\operatorname{dim} \mathcal{N}=n-2$.

Теорема 3. Для любых строго перспективных реперов $\mathcal{R}$ и $\mathcal{R}^{\prime}$ плоскость $\mathcal{L}_{\left(\mathcal{R}, \mathcal{R}^{\prime}\right)}$ является гиперплоскостью в $\mathbb{P}_{n}$.

Доказательство. В случае $n=2$ утверждение совпадает с классической теоремой Дезарга (см., например, [3]). Далее будем полагать $n>2$.

Покажем, что $\operatorname{dim} \mathcal{L}_{\left(\mathcal{R}, \mathcal{R}^{\prime}\right)} \geqslant n-1$. Для этого убедимся в том, что $B_{1} \notin \mathcal{N}$. В самом деле, если предположить, что $B_{1} \in \mathcal{N}$, то $B_{1} \in \mathcal{M}$. С учетом $B_{1} \in A_{1} E$ и $A_{1} \in \mathcal{M}$ мы приходим к тому, что $E \in \mathcal{M}$, что невозможно, так как $E$ - единичная точка репера $\mathcal{R}$. Заметим, что аналогичным образом можно убедиться в том, что $B_{j} \notin \mathcal{N}(j=\overline{2, n})$.

Покажем теперь, что $\operatorname{dim} \mathcal{L}_{\left(\mathcal{R}, \mathcal{R}^{\prime}\right)} \leqslant n-1$, т.е. что $B_{j} \in\left\langle B_{1}, \mathcal{N}\right\rangle$ для любого $j=\overline{2, n}$. Действительно, рассмотрим 3-плоскость $\left\langle A_{0} A_{1}, A_{0} A_{j}, A_{0} E\right\rangle$. Она содержит 2-плоскости $\left\langle A_{1}, A_{j}, E\right\rangle$ и $\left\langle A_{1}^{\prime}, A_{j}^{\prime}, E^{\prime}\right\rangle$. Пересечение этих 2-плоскостей представляет собой прямую, содержащую точки $C_{j}$, $B_{i}$ и $B_{j}$, где $C_{j}=A_{1} A_{j} \cap A_{1}^{\prime} A_{j}^{\prime}$. Значит, точка $B_{j}$ лежит на прямой $B_{1} C_{j}$ для любого $2 \leqslant j \leqslant n$. Тогда $B_{j} \in\left\langle B_{1}, \mathcal{N}\right\rangle$, что и требовалось доказать.

Замечание 3. Неравенство $\operatorname{dim} \mathcal{L}_{\left(\mathcal{R}, \mathcal{R}^{\prime}\right)} \leqslant n-1$ также непосредственно вытекает из результатов работы [8].

Определение 8. Назовем $\mathcal{L}_{\left(\mathcal{R}, \mathcal{R}^{\prime}\right)}$ гиперплоскостью Дезарга, порожденной реперами $\mathcal{R}$ и $\mathcal{R}^{\prime}$.

Теорема 4. Пусть $\mathcal{R}$ и $\mathcal{R}^{\prime}$ - строго перспективные реперы $n$-мерного проективного пространства $\mathbb{P}_{n}$ с иентром $A$. Тогда они эквивалентны в том и только в том случае, когда гиперплоскость Дезарга $\mathcal{L}_{\left(\mathcal{R}, \mathcal{R}^{\prime}\right)}$ проходит через чентр.

Доказательство. Пусть реперы $\mathcal{R}$ и $\mathcal{R}^{\prime}$ строго перспективны. Тогда они перспективны, и в силу утверждения 4 для них справедливы равенства (6). Пусть $\left(x^{0}: x^{1}: \ldots: x^{n}\right)$ - однородные координаты на $\mathbb{P}_{n}$ относительно репера $\mathcal{R}$. В этих координатах гиперплоскости $\mathcal{M}$ и $\mathcal{M}^{\prime}$ задаются соответственно уравнениями

$$
\mathcal{M}: x^{0}=0, \quad \mathcal{M}^{\prime}: a_{i} x^{i}-h x^{0}=0 .
$$


Таким образом, уравнение пучка $S$ гиперплоскостей $S_{(\lambda: \mu)}$, проходящих через плоскость $\mathcal{N}$, можно представить в виде

$$
S_{(\lambda: \mu)}: \lambda x^{0}+\mu a_{i} x^{i}=0, \quad \lambda^{2}+\mu^{2} \neq 0 .
$$

Для точки $B_{1}$ найдется такой вектор $\boldsymbol{B}_{1} \in \pi^{-1}\left(B_{1}\right)$, что

где

$$
\boldsymbol{B}_{1}=-\frac{e}{a_{1}} \boldsymbol{A}_{1}+\boldsymbol{E}
$$

$$
e=a_{1}+\ldots+a_{n}+1-h .
$$

Гиперплоскость $\mathcal{L}_{\left(\mathcal{R}, \mathcal{R}^{\prime}\right)}$ выделяется из данного пучка условием $B_{1} \in \mathcal{L}_{\left(\mathcal{R}, \mathcal{R}^{\prime}\right)}$, которое, в свою очередь, равносильно соотношению

$$
\lambda=(1-h) \mu .
$$

Подставляя его в $(7)$, получим уравнение гиперплоскости $\mathcal{L}_{\left(\mathcal{R}, \mathcal{R}^{\prime}\right)}$ :

$$
\mathcal{L}_{\left(\mathcal{R}, \mathcal{R}^{\prime}\right)}:(1-h) x^{0}+a_{i} x^{i}=0, \quad \lambda^{2}+\mu^{2} \neq 0 .
$$

Таким образом,

$$
A_{0} \in \mathcal{L}_{\left(\mathcal{R}, \mathcal{R}^{\prime}\right)} \stackrel{(8)}{\Longleftrightarrow} h=1 \stackrel{\text { теорема } 2}{\Longleftrightarrow} \mathcal{R} \sim \mathcal{R}^{\prime} .
$$

5. Правые действия матричных групп на многообразиях $\mathcal{F}\left(\mathbb{P}_{n}\right)$ и $\mathcal{F}(V)$. Опишем действия матричных групп на реперах и базисах, поскольку именно матричные группы обычно выступают в роли структурных групп главных расслоений (см., например, конструкции расслоений в [4]).

Рассмотрим два адаптированных репера $\mathcal{R}$ и $\mathcal{R}^{\prime}$, и пусть формула перехода от первого ко второму имеет вид (4). Из коэффициентов данной формулы составим матрицу

$$
A=\left(\begin{array}{cccc}
1 & \alpha_{1} & \cdots & \alpha_{n} \\
0 & \alpha_{1}^{1} & \cdots & \alpha_{n}^{1} \\
\vdots & \vdots & \ddots & \vdots \\
0 & \alpha_{1}^{n} & \cdots & \alpha_{n}^{n}
\end{array}\right)=\left(\begin{array}{cc}
1 & \alpha \\
0 & A_{0}
\end{array}\right)
$$

где $A_{0} \in \mathbf{G L}(n), \alpha$-строка из $n$ элементов. Хорошо известно (см. [4]), что множество всех невырожденных матриц вида (9) образует группу $\mathbf{G A}(n)$. Ее важнейшими подгруппами являются (1) группа $\mathbf{G A}_{0}(n)$, образованная матрицами вида $\left(\begin{array}{cc}1 & 0 \\ 0 & A_{0}\end{array}\right)$,изоморфная полной линейной группе
$\mathbf{G L}(n) ;$ (2) группа $\mathbf{T}(n)$, образованная матрицами вида $\left(\begin{array}{ll}1 & \alpha \\ 0 & \mathbb{I}_{n}\end{array}\right)$,изоморфная аддитивной группе $\mathbb{R}^{n}$
(здесь $\mathbb{I}_{n}-$ единичная матрица порядка $\left.n\right)$.

Будем рассматривать (4) как формулу действия матрицы $A$ на репер $\mathcal{R}$, а репер $\mathcal{R}^{\prime}$ - соответственно как образ репера $\mathcal{R}$ по данному действию:

$$
\mathcal{R}^{\prime}=\mathcal{R} \cdot A .
$$

При этом для любых базисов $\boldsymbol{R}$ и $\boldsymbol{R}^{\prime}$, порождающих реперы $\mathcal{R}$ и $\mathcal{R}^{\prime}$ соответственно, найдется скалярный множитель $\lambda \neq 0$ такой, что

$$
\lambda \boldsymbol{\mathcal { R }}^{\prime}=\boldsymbol{R} \cdot A,
$$

где справа стоит обычное произведение матрицы-строки из элементов базиса $\boldsymbol{R}$ на матрицу $A$.

Аналогично, действие матрицы $A_{0} \in \mathbf{G} \mathbf{L}(n)$ на многообразии $\mathcal{F}(V)$ можно задать по формуле

$$
\mathcal{B}^{\prime}=\mathcal{B} \cdot A_{0}, \quad \mathcal{B}, \mathcal{B}^{\prime} \in \mathcal{F}(V) .
$$

Формула (10) задает правое действие матричной группы $\mathbf{G A}(n)$ на многообразии $\mathcal{F}\left(\mathbb{P}_{n}\right)$, а формула (11) - правое действие группы $\mathbf{G L}(n)$ на $\mathcal{F}(V)$.

Рассмотрим гомоморфизм групп Ли

$$
\gamma: \mathbf{G A}(n) \rightarrow \mathbf{G L}(n), \quad A \mapsto A_{0},
$$


где матрица $A$ имеет строение (9). Его ядром является $\mathbf{T}(n)$, причем факторгруппа по ядру изоморфна $\mathbf{G L}(n)$.

Если в (10) матрица $A$ принадлежит $\mathbf{T}(n)$, то формула перехода между реперами $\mathcal{R}$ и $\mathcal{R}^{\prime}$ имеет вид $(5)$, а потому $\mathbf{T}(n)$-орбита любого репера $\mathcal{R} \in \mathcal{F}\left(\mathbb{P}_{n}\right)$ совпадает с его $H$-орбитой, т.е. $\mathcal{F}\left(\mathbb{P}_{n}\right) / \mathbf{T}(n)=\mathcal{Q}\left(\mathbb{P}_{n}\right)$.

Утверждение 5. Группа $\mathbf{G L}(n)$ действует справа на пространстве $\mathbf{T}(n)$-орбит $\mathcal{Q}\left(\mathbb{P}_{n}\right)$ по правилу

$$
[\mathcal{R}] \cdot A_{0}=[\mathcal{R} \cdot A], \quad A \in \gamma^{-1}\left(A_{0}\right) .
$$

Многообразия $\mathcal{Q}\left(\mathbb{P}_{n}\right)$ и $\mathcal{F}(V)$ изоморфны как $\mathbf{G L}(n)$-пространства, причем $\bar{\alpha}$-соответствующий изоморфизм.

\section{СПИСОК ЛИТЕРАТУРЫ}

1. Белова О. О. Связности в расслоениях, ассоциированных с многообразием Грассмана и пространством центрированных плоскостей// Фундам. прикл. мат. - 2008. - 14, № 2. - С. 29-67.

2. Берже М. Геометрия. - М.: Мир, 1984.

3. Ефимов Н. В. Высшая геометрия. - М.: Физматлит, 2004.

4. Кобаяси ШІ., Номидзу К. Основы дифференциальной геометрии. - М.: Наука, 1981.

5. Омельян О. М., Шевченко Ю. И. Редукции объекта центропроективной связности и тензора аффинного кручения на распределении плоскостей// Мат. заметки. - 2008. - 84, № 1. - С. 99-107.

6. Полякова K. В. Параллельные перенесения на поверхности проективного пространства// Фундам. прикл. мат. - 2008. - 14, № 2. - С. 129-177.

7. Akivis A., Goldberg V. Projective Differential Geometry of Submanifolds. - Amsterdam: North-Holland, 1993.

8. Bell P. O. Generalized theorems of Desargues for $n$-dimensional projective space// Proc. Am. Math. Soc. - 1955. - № 6. - P. 675-681.

9. Cartan E. La théorie de groupes finis et continus et la géométrie différentielle traitées par la méthode di repère mobile. - Paris: Gauthier-Villars, 1937.

10. Conlon L. Differential Manifolds: A First Course. — Boston: Birkhäuser, 1993.

11. Ivey T. A., Landsberg J. M. Cartan for Beginners: Differential Geometry via Moving Frames and Exterior Differential Systems. - Providence, Rhode Island: Am. Math. Soc., 2003.

Кулешов Артур Владимирович

Балтийский федеральный университет им. И. Канта, Калининград

E-mail: arturkuleshov@yandex.ru 